Fukushima J. Med. Sci.,

Vol. 60, No. 2, 2014

[Original Article]

\title{
EFFICACY OF 2-PORT LAPAROSCOPIC SURGERY FOR GYNECOLOGIC DISEASE : INVENTION OF NEW SUTURE TECHNIQUE
}

\author{
SATOSHI SUZUKI ${ }^{1)}$, SHIGENORI FURUKAWA ${ }^{1)}$, MIKI OHARA ${ }^{1)}$, KIYOSHI KANNO ${ }^{2)}$, \\ SHUN YASUDA $^{1)}$, TAKEHARU OHZEKI ${ }^{3)}$, TAKAFUMI WATANABE ${ }^{1)}$, \\ HIDENORI TAKAHASHI ${ }^{4)}$, HIROSHI SUZUKI ${ }^{2)}$ and KEIYA FUJIMORI ${ }^{1)}$ \\ ${ }^{1)}$ Department of Obstetrics and Gynecology, Fukushima Medical University School of Medicine, Fuku- \\ shima, Japan, ${ }^{2)}$ Department of Obstetrics and Gynecology, Jusendo General Hospital, Koriyama, Japan, \\ ${ }^{3)}$ Department of Obstetrics and Gynecology, Takeda General Hospital, Aizuwakamatsu, Japan, ${ }^{4)}$ Castle \\ Bell Clinic, Nagoya, Japan
}

(Received April 9, 2014, accepted October 9, 2014)

\begin{abstract}
Background and Objective : To evaluate the efficacy and safety of 2-port laparoscopic surgery for ovarian tumors and uterine leiomyomas. A conventional slip knot under laparoscopic surgery is useful, but it is difficult for beginners. Therefore, we developed new suture technique.

Methods : Between January 2011 and December 2012, 38 patients underwent 2-port total laparoscopic cystectomy (TLC) ; between January 2010 and December 2011, 45 patients underwent multiport (3 or 4 ports) TLC. Between January 2011 and December 2012, 25 patients underwent 2-port laparoscopic myomectomy (LM) ; between January 2010 and December 2011, 34 patients underwent multiport (3 or 4 ports) LM. Surgery time, blood loss, postoperative length of stay and complications were retrospectively compared in each group.

Results : No significant differences in surgery time, blood loss or postoperative length of stay were found between 2-port TLC and multiport TLC. No significant differences were found in the aforementioned parameters between 2-port LM and multiport LM. We showed here a new suture technique 'intra-abdominal suturing/extra-abdominal traction method' instead of conventional slip knot. Conclusions: We confirmed that 2-port TLC and LM are less invasive than conventional multiport laparoscopic surgery ; furthermore, they are as safe as the conventional procedure. The new suture technique is easier than conventional slip knot and can applies sufficient tension to the suture knot for beginners.
\end{abstract}

Key words : 2-port laparoscopic surgery, myomectomy, cystectomy, suture technique

\section{INTRODUCTION}

Laparoscopic surgery is currently widely used in gynecologic procedures, and even less invasive techniques are being requested. Single-port laparoscopic surgery is aesthetically superior to 3-port and 4-port (multiport) laparoscopic surgery ${ }^{1}$. However, its indications are limited because of interference between the forceps and scope as well as other technical difficulties. Two-port laparoscopic surgery reduces forceps interference, makes suturing easier, and is indicated in a wider number of cas- es. We began performing 2-port laparoscopic surgery at our institution in 2010, and it has been a standard procedure since 2011. In this study, we compared patients' characteristics (age, body weight, BMI, maximum tumor size), surgery time, blood loss, postoperative length of stay and complications which prolonged hospital stay, between 2-port laparoscopic ovarian cystectomy (2-port TLC) and 2 -port laparoscopic myomectomy (2-port LM) with multiport laparoscopic surgery before and just after introduction of 2-port laparoscopy at our institution. Furthermore, we introduce new suture tech-

Corresponding author: Satoshi Suzuki Email: ss3104@fmu.ac.jp

https://www.jstage.jst.go.jp/browse/fms http://www.fmu.ac.jp/home/lib/F-igaku/ 
nique 'intra-abdominal suturing/extra-abdominal traction method'.

\section{SUBJECTS AND METHODS}

This study included patients who underwent surgery at Fukushima Medical University Hospital, including : 2-port TLC in 38 patients and 2-port LM in 25 patients between January 2011 and December 2012 ; multiport TLC in 45 patients and multiport LM in 34 patients between January 2010 and December 2011. Surgery was performed with pneumoperitoneum under general anesthesia in all cases.

For multiport laparoscopy, the first trocar was inserted by an open technique above the umbilicus, the second and third trocars were inserted in the left and right lateral regions. For 2-port laparoscopy, a $2.5 \mathrm{~cm}$ longitudinal incision in the umbilical ring and insertion by an open technique of a $5 \mathrm{~mm}$ Xcel Long $^{\circledR}$ (Johnson and Johnson, NJ, USA) and E.Z trocar $5 \mathrm{~mm}$ short $^{\circledast}$ (Hakko Co., Ltd., Nagano, Japan) (multiple trocar technique: Fig. 1a); or placement of a Lap-Protector ${ }^{\mathrm{TM}}$ (Hakko Co., Ltd., Nagano, Japan) in an umbilical incision and mounting of an E.Z Access ${ }^{\mathrm{TM}}$ with insertion of two $5 \mathrm{~mm}$ trocars $(\mathrm{E} \cdot \mathrm{Z}$ Access technique : Fig. 1b) was used. Third trocar was inserted in the left lateral region. In all LM case, vasopressin was locally injected at myometrium. In the multiple trocar technique, the specimen was removed through the third trocar in the left lateral region. In the $\mathrm{E} \cdot \mathrm{Z}$ Access technique, the specimen was removed through the Lap-Protector in the umbilicus. For multiport and multiple-trocar LM, a Gynecare laparoscopic morcellator (Johnson and Johnson, NJ, USA) was used. Surgery in all patients was performed by three surgeons with experience in at least 100 cases. We obtained informed consent from each patient in photos.

\section{Statistical analysis}

Statistical analysis was accomplished using the

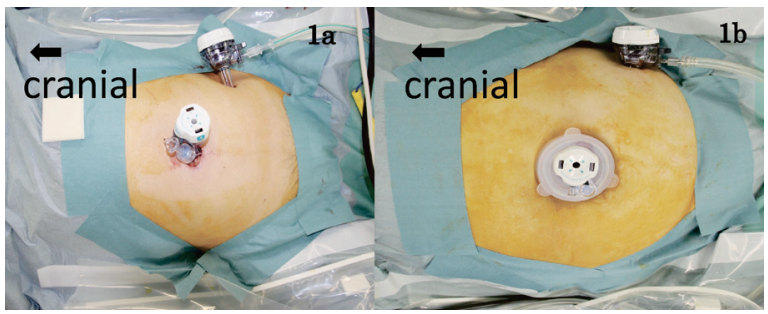

Fig. 1. Trocar arrangement of the multiple trocar technique (a). Trocar arrangement of the EZ access technique (b).
SPSS ver. 21 (IBM, NY, USA). The t-test was used as indicated. All data were presented as mean \pm SD. All $\mathrm{P}$ values were 2 -sided. A P value of 0.05 was considered statistically significant.

\section{RESULTS}

Table 1a and Table 2a show the characteristics (mean age, body weight, BMI and maximum tumor size) of patients who underwent TLC or LM. Table $1 \mathrm{~b}$ and Table $2 \mathrm{~b}$ shows surgery time, bleeding, postoperative length of stay and complications of patients.

(1) Comparison of TLC cases (Table 1)

Patients' characteristics were not significantly different. Surgery time, bleeding, postoperative length of stay were not significantly different. There was no complication which lead to longer hospitalization in both groups.

(2) Comparison of LM cases (Table 2)

Age, body weight and BMI were not significantly different, but maximum tumor size was significantly large in multiport group. Surgery time tended to be longer in the 2-port LM group ; however, the difference was not significant $(p=0.160)$. Blood loss tended to be decreased in the 2-port LM group ; however, the difference was not significant $(p=$ 0.055). Postoperative length of stay was not significantly different. There were 3 complications in multiport LM group (massive hemorrhage, convert

Table 1a. Patients' characteristics who underwent TLC (total laparoscopic cystectomy)

\begin{tabular}{lccc}
\hline & multiport & 2-port & p-value \\
\hline No. of cases & 45 & 38 & \\
Age & $34.9 \pm 9.3$ & $33.4 \pm 8.0$ & n.s. \\
Body Weight $(\mathrm{kg})$ & $55.3 \pm 10.9$ & $54.0 \pm 10.5$ & n.s. \\
BMI & $22.2 \pm 4.3$ & $21.5 \pm 3.5$ & n.s. \\
Tumor size $(\mathrm{cm})$ & $6.8 \pm 1.6$ & $6.3 \pm 1.9$ & n.s. \\
\hline
\end{tabular}

Table 1b. Performance of surgery and complications

\begin{tabular}{|c|c|c|c|}
\hline & multiport & 2 -port & $\mathrm{p}$-value \\
\hline No. of cases & 45 & 38 & \\
\hline Surgery time (min) & $118.7 \pm 52.8$ & $137.1 \pm 53.1$ & n.s. \\
\hline Bleeding (ml) & $46.7 \pm 71.8$ & $68.2 \pm 197.3$ & n.s. \\
\hline $\begin{array}{l}\text { Postoperative length } \\
\text { of stay (day) }\end{array}$ & $4.9 \pm 1.4$ & $4.7 \pm 1.4$ & n.s. \\
\hline $\begin{array}{l}\text { Complication (No of } \\
\text { case) }\end{array}$ & 0 & 0 & \\
\hline
\end{tabular}


Table 2a. Patients' characteristics who underwent LM (laparoscopic myomectomy)

\begin{tabular}{lccc}
\hline & multiport & 2-port & p-value \\
\hline No. of cases & 34 & 25 & \\
Age & $35.9 \pm 5.6$ & $32.9 \pm 7.7$ & n.s. \\
Body Weight $(\mathrm{kg})$ & $60.4 \pm 10.2$ & $55.7 \pm 7.6$ & n.s. \\
BMI & $23.6 \pm 4.1$ & $22.1 \pm 2.8$ & n.s. \\
Tumor size $(\mathrm{cm})$ & $7.3 \pm 1.8$ & $6.0 \pm 2.6$ & 0.026 \\
\hline
\end{tabular}

Table 2b. Performance of surgery and complications

\begin{tabular}{|c|c|c|c|}
\hline & multiport & 2 -port & p-value \\
\hline No. of cases & 34 & 25 & \\
\hline Surgery time (min) & $165.4 \pm 72.4$ & $191.9 \pm 67.8$ & n.s. $(0.160)$ \\
\hline Bleeding (ml) & $379.8 \pm 553.0$ & $174.1 \pm 221.1$ & n.s. $(0.055)$ \\
\hline $\begin{array}{l}\text { Postoperative length } \\
\text { of stay (day) }\end{array}$ & $5.8 \pm 1.9$ & $5.2 \pm 1.2$ & n.s. $(0.119)$ \\
\hline $\begin{array}{l}\text { Complication (No of } \\
\text { case) }\end{array}$ & 1 & 1 & \\
\hline
\end{tabular}

to laparotomy, damage to intestine), and one complication in 2-port LM group (subcutaneous abscess at the umbilicus).

\section{(3) New suture technique}

We invented 'intra-abdominal suturing/extraabdominal traction method' and frequently use this technique. This is performed in the following manner (Fig. 2a-2e). [a] Insert a needle with $70 \mathrm{~cm}$ absorbable suture into abdominal cavity through the $12 \mathrm{~mm}$ trocar and stitch the myometrium without cutting the suture ; $[\mathrm{b}]$ The end with the needle at- tached is pulled outside the body (long tail), and the other end of the suture is the short tail ; [c] To make a Surgeon's knot, wrap the long tail of the suture around the right hand forceps and pull the short tail with right hand forceps ; and [d, e] Traction is applied to the short tail with right hand forceps in abdominal cavity, and extracorporeal traction is applied to the long tail using the left hand. 2nd and 3rd ligation was similarly performed in abdominal cavity but traction to the long tail was applied by left hand forceps.

\section{DISCUSSION}

Laparoscopic surgery is currently widely used in gynecologic procedures, and even less invasive techniques are being requested. Since single-port laparoscopic appendectomy was reported by Pelosi et al. ${ }^{1}{ }^{1}$ in 1992, attention has focused on single-port and 2-port laparoscopy for reduced port surgery. Single-port laparoscopy is advantageous because it is aesthetically superior to conventional multiport laparoscopy ${ }^{2}$. However, it has limitations not found in multiport laparoscopy, including greater interference between the forceps and scope, and a narrower surgical field. In addition, although no significant differences in surgery time, blood loss and complications have been reported in some procedures ${ }^{3,4)}$, longer surgery times and greater blood loss are a concern for surgeons not experienced in single-port techniques or laparoscopic surgery novices. These concerns can be overcome to some extent by sufficient training and clinical experience ${ }^{5}$. However,
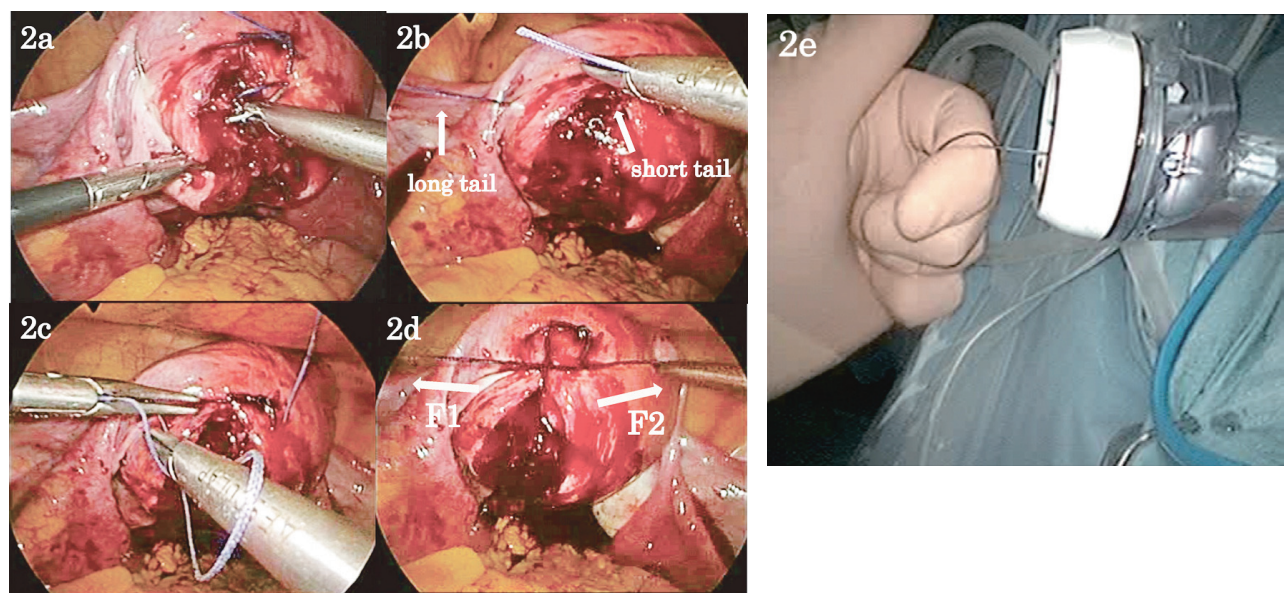

Fig. 2. Insert a needle with a $70 \mathrm{~cm}$ absorbable suture into the abdominal cavity without cutting the suture, and stitch the myometrium (a). Pull the needle outside the body (this side is long tail), and adjust the other end of the suture to be about 2 to $3 \mathrm{~cm}$ (short tail) (b). Wrap the long tail of the suture around the right hand forceps and pull the short tail with the right hand forceps and make a knot (c). In the abdominal cavity, pull the short tail toward F2 with the right hand forceps. Outside the abdominal cavity, draw the long tail toward F1 with the left hand $(\mathrm{d}, \mathrm{e})$. 
Table 3. Merit and demerit of 2-port laparoscopic surgery

\begin{tabular}{|c|c|}
\hline \multicolumn{2}{|c|}{ Merit and demerit of 2-port laparoscopic surgery } \\
\hline Merit & Demerit \\
\hline $\begin{array}{l}\text { - Wide application in sur- } \\
\text { gery } \\
\text { - Fewer incisions (compared } \\
\text { with multiport surgery) } \\
\text { - Easier than single port } \\
\text { surgery } \\
\text { - Surgeon can do surgery } \\
\text { with easy posture }\end{array}$ & $\begin{array}{l}\text { - Interference between the } \\
\text { forceps and the scope } \\
\text { - Larger navel wound No } \\
\text { assistance by an assistant } \\
\text { surgeon } \\
\text { - Educational problem for } \\
\text { assistant }\end{array}$ \\
\hline
\end{tabular}

in general, single-port laparoscopy is difficult for newcomers or surgeons with less experience in laparoscopic surgery ${ }^{6)}$; in addition, surgeons who can perform single-port laparoscopic surgery and its indications are limited. In fact, the Japan Society of Gynecologic and Obstetric Endoscopy and Minimally Therapy (JSOGE) recommends that "To safely perform single-port laparoscopic surgery, it should only be used after formal training in laparoscopic surgery and only by those skilled in specific surgical procedures." ${ }^{7)}$ However, in 2-port laparoscopy, the addition of just one trocar in the lateral region can enable manipulations comparable to conventional multiport laparoscopic surgery. This provides many advantages to both patients and surgeons, and thus we began performing 2-port laparoscopic surgery in our department.

Table 3 lists some of the advantages and disadvantages of 2-port laparoscopic surgery compared to single-port and multiport laparoscopic surgery. Insertion of a third trocar in the left lateral region reduces forceps interference and we can markedly freely perform surgical techniques such as suturing. Application to many types of surgery is possible, and as mentioned previously, we now use 2-port laparoscopic surgery as a standard laparoscopic procedure in our department. Moreover, compared to conventional diamond trocar configuration (3-port laparoscopic surgery), the surgeon does not have to stand as if hugging the patient to the right side (when the surgeon is standing to the left of the patient), but can perform surgery in a more upright stance. This lessens the burden and stress during surgery on short surgeons and female physicians (Fig. 3a, 3b).

A disadvantage of 2-port laparoscopic surgery is that because of a $2.5 \mathrm{~cm}$ incision in the umbilicus, attention must be paid to postoperative aesthetic appearance, particularly in patients with a shallow umbilicus. In addition, interference between the scope

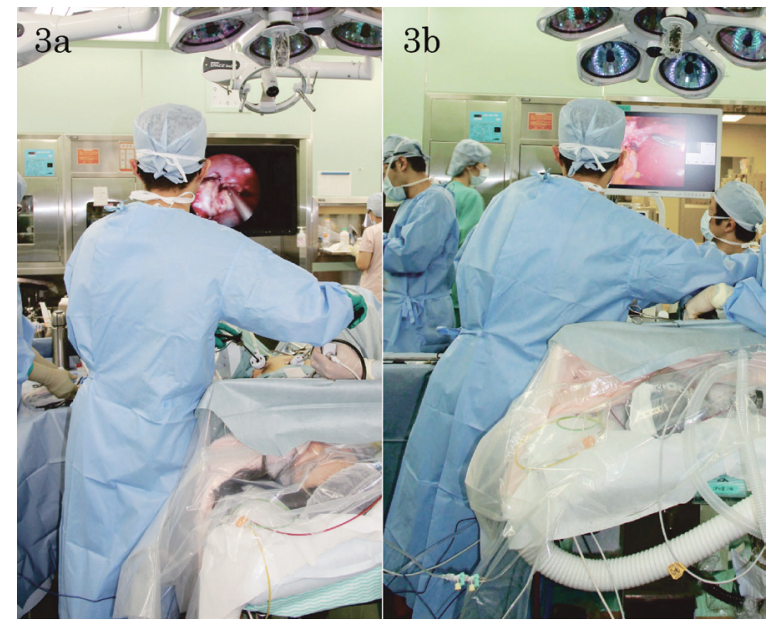

Fig. 3. Posture of surgeon in 2-port laparoscopic surgery (a). Posture of surgeon in conventional multiport surgery (b). Surgeons can perform surgery in a more upright stance in 2-port laparoscopic surgery, and feel less fatigue during long surgery $(a, b)$.

and right-hand forceps can occur, but this is less than with single-port laparoscopy and can be resolved by optimal camera operation by surgical assistant. Because no assistance can be provided by a surgical assistant, great care must be taken in patients with abundant visceral fat both to secure the surgical field and artifices are need to suture tissue under tension such as the myometrium.

A slip knot may be used for suturing tension sites so that the suture thread does not become loose. Because slip knot is difficult for beginners, so we invented 'intra-abdominal suturing/extra-abdominal traction method'. We frequently use this technique together with slip knot technique. This method applies sufficient tension to the suture knot and enables suturing without assistance from an assistant even at high-tension sites such as the myometrium without the suture loosening. By this method, the suture can be used repeatedly about 3 or 4 times and it is economical.

Various techniques such as the foregoing facilitate the performance of several types of surgery such as LM, compared to single-port laparoscopic surgery. However, the assistant only handles the camera ; the right hand, unlike conventional procedures, is not used to expose the surgical field, grasp tissue, or cut sutures. Thus, education for assistants still remains an issue.

In conducting this study, because 2-port laparoscopic surgery was performed without an assistant, increased time for myoma enucleation and suturing in LM and increased blood loss were potential con- 
cerns. However, we found that, compared with conventional multiport laparoscopic surgery, surgery time and blood loss were not significantly increased. On the contrary, blood loss in multiport LM tended to be higher, although the difference was not significant. In addition to the fact that 2-port laparoscopic surgery was not technically limited and not inferior to multiport laparoscopic surgery in terms of manipulation, selection bias may also have had an influence ; specifically, multiport laparoscopic was selected initially in patients with large or multiple leiomyomas in whom difficult enucleation was anticipated. Although not significant, postoperative length of stay tended to be shorter in both 2-port TLC and 2-port LM and there were few complications which prolong hospitalization. In this study we didn't examine the post-operative pain, pain from umbilicus incision wound and conventional multiport wound is equivalent ${ }^{8)}$. Therefore, 2-port laparoscopy considered to be less invasive (smaller number of wound) than conventional multiport laparoscopic surgery and safety is equivalent.

Our department introduced 2-port laparoscopy 4 years ago, and no significant disadvantages to patients in terms of surgery time, blood loss, or other complications have been observed to date. Table 3 shows the substantial advantages of 2-port laparoscopy. Moreover, because our institution is a university hospital, surgeons with various levels of experience, from board-certified surgeons to first-year residents, perform laparoscopic surgery. Thus, 2 -port laparoscopy, which is both easy to perform and is minimally invasive, is a suitable surgical procedure from an educational perspective. We now use 2-port laparoscopy as a standard procedure and are planning a further evaluation of its usefulness on a larger number of patients.

\section{CONCLUSION}

2-prot laparoscopy is less invasive (smaller number of wound), easier and safe surgical procedure, so it is beneficial for both patients and physicians.

\section{ACKNOWLEDGEMENT : NONE}

Disclosure : We have no relationship with companies that may have a financial interest.

\section{REFERENCES}

1. Pelosi MA, Pelosi MA 3rd. Laparoscopic appendectomy using a single umbilical puncture (minilaparoscopy). J Reprod Med, 37(7) : 588-594, 1992.

2. Shepherd JA, King S, Badia CD, et al. Single Port Access $\left(\mathrm{SPA}^{\mathrm{TM}}\right)$ Hysterectomy in a Teaching Institution: Comparison of single Port to traditional Multiport Laparoscopy. Abstracts/ Journal of minimally Invasive Gynecology, 15 : S87, 2008. DOI:http://dx.doi.org/10.1016/j.jmig.2008.09.334

3. Lee M, Kim SW, Nam EJ, Yim GW, Kim S, Kim YT. Single-port laparoscopic surgery is applicable to most gynecologic surgery : a single surgeon's experience. Surg Endosc, 26(5) : 1318-1324, 2012.

4. Behnia-Willison F, Foroughinia L, Sina M, McChesney P. Single incision laparoscopic surgery(SILS) in gynaecology : feasibility and operative outcomes. Aust N Z J Obstet Gynaecol, 52(4) : 366-370, 2012.

5. Han CM, Lee CL, Su H, Wu PJ, Wang CJ, Yen CF. Single-port laparoscopic myomectomy : initial operative experience and comparative outcome. Arch Gynecol Obstet, 287(2) : 295-300, 2013.

6. Hirano K, Kunimi K, Keyama K, Nakayama A. Study on acquirement of the single-incision laparoscopic surgery technique. Modern trends in obstetrics and gynecology, 59(29): 187-190, 2010.

7. Japan Society of Gynecologic and Obstetric Endoscopy and Minimally Invasive Therapy (JSGOE) : http://www.asas.or.jp/jses/

8. Emre TK, Ilhan E, Yakan S, Cengiz F, Senlikci A, Aktürk HE. Single-port laparoscopic cholecystectomy versus the classical four port laparoscopic cholecystectomy : a randomized prospective clinical trial. Minerva Chir, 69(1) : 1-7, 2014. 